\title{
WHAT ARTIFICIAL INTELLIGENCE REVEAL ABOUT THE BARRIERS HISPANICS/LATINOS FACE ACCESSING DEPRESSION TREATMENT?
}

\author{
Ruby Castilla-Puentes, MD,DrPH,MBA (1,2,3); Dinorah Villanueva MS, MBA, (2, 3); Tatiana Falcone, MD,MPH (4); \\ Laura Jimenez-Parrado,MD (5); Liliana. Gil Valleta (6), Caroline Brethenoux. (6).
}

(1)Center for Public Health Practice- Drexel University, Philadelphia, PA, USA; (2) Neuroscience- Janssen Research \& Development, LLC, Titusville, NJ, USA; (3) Hispanic Organization of Leadership and Achievement- HOLA- Johnson \& Johnson, Employee Resource Group, New Brunswick, NJ, USA; (4) Cleveland Clinic Lerner College of Medicine- Cleveland, Ohio- USA, Department of Psychiatry/Epilepsy, Cleveland, USA; (5) Investigation Group - Sleep Disorders and Forensic Psychiatry - Universidad Nacional de Colombia, Bogota, Colombia; (6) CulturIntel Inc, New York City- New York- USA

Background: Hispanics in the US, utilize less mental health resources, including depression treatment vs. the general population. This is the first-time that advanced data extraction techniques and leading artificial intelligence (AI) - powered algorithms - are used to better understand the Hispanics patients and their journey to seek medical attention.

Methods: We analyzed 12 months of digital discussions about depression to identify patterns in the topics and barriers that correlate to dealing with depression and seeking help and/or treatment. The search was limited to conversations originating from USA internet protocol addresses and tagged based on self-identified profile descriptions. Advanced search techniques, including web spiders, crawlers, and site scraping were applied using Culturlntel Al powered algorithms. All data was extracted and tagged by topics and a large, unstructured 'big' dataset was created.

Results: A total of 543,000 discussions about depression were analyzed ( 43,000 Hispanics, $~ 398,000$ non-Hispanics). Hispanics concern toward social appearances weighs on the subject; "stigma" is discussed nearly twice as much, while the toll on others is higher than the non-Hispanic population. Hispanics were significantly more resigned (hopeless/helpless), less involved, and come into diagnosis with a struggling mindset/ attitude at a 2.6x greater rate. Cultural beliefs related to their "hopelessness," play a negative factor to care that is 1.5 times greater at the stage of pre-diagnosis/ suspect.

Conclusion: "Resigned", "hopelessness/helplessness", "learned helplessness" concepts prevent patients from seeking help as they rather "live and cope with it" than face public/ social shame or labeling. Our findings suggest a greater need to proactively educate and empower caregivers, health care professionals and family with culture sensitive information to contextualize depression. Al powered data analysis can contribute to better healthcare communications and patient engagement.
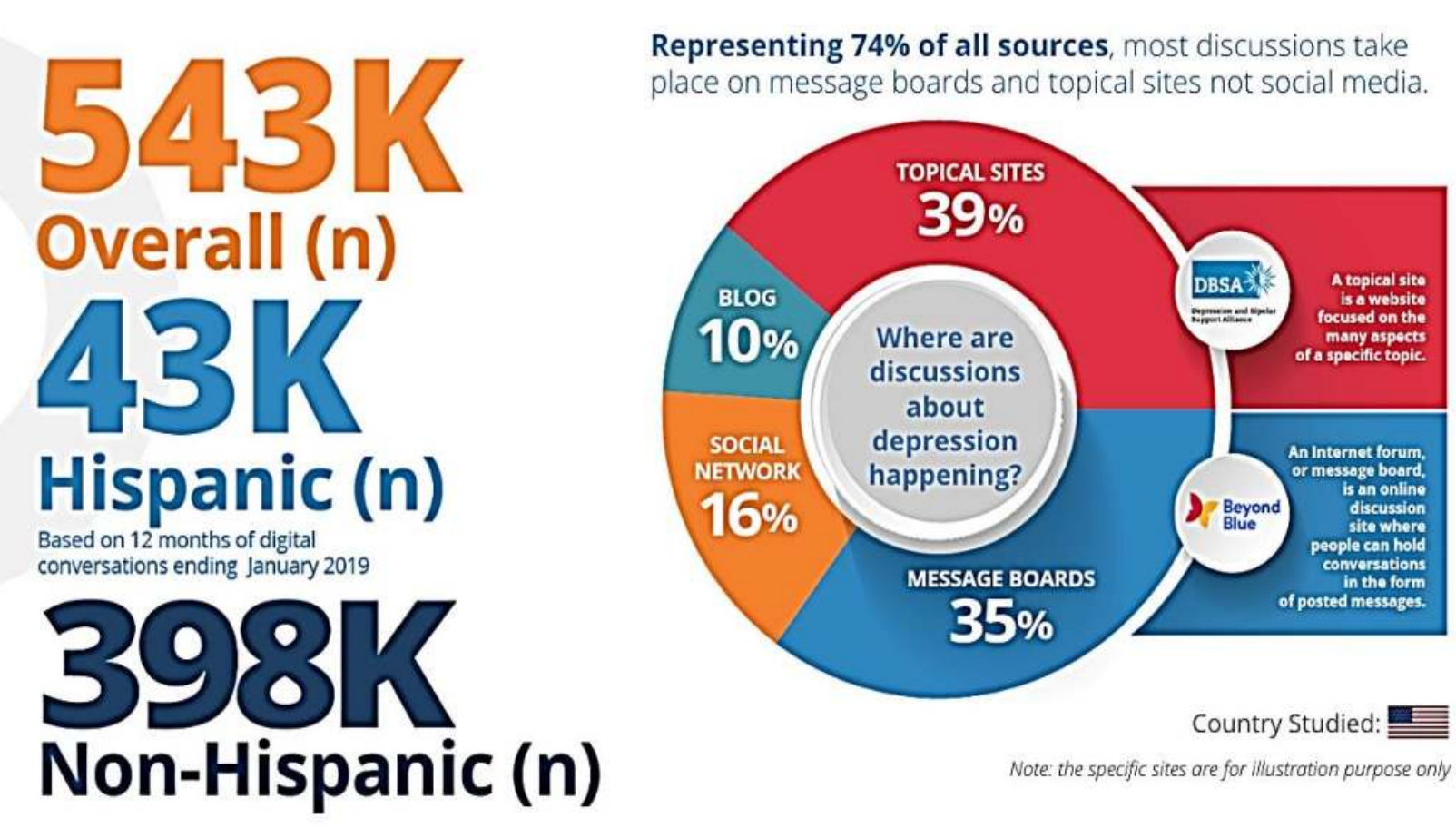

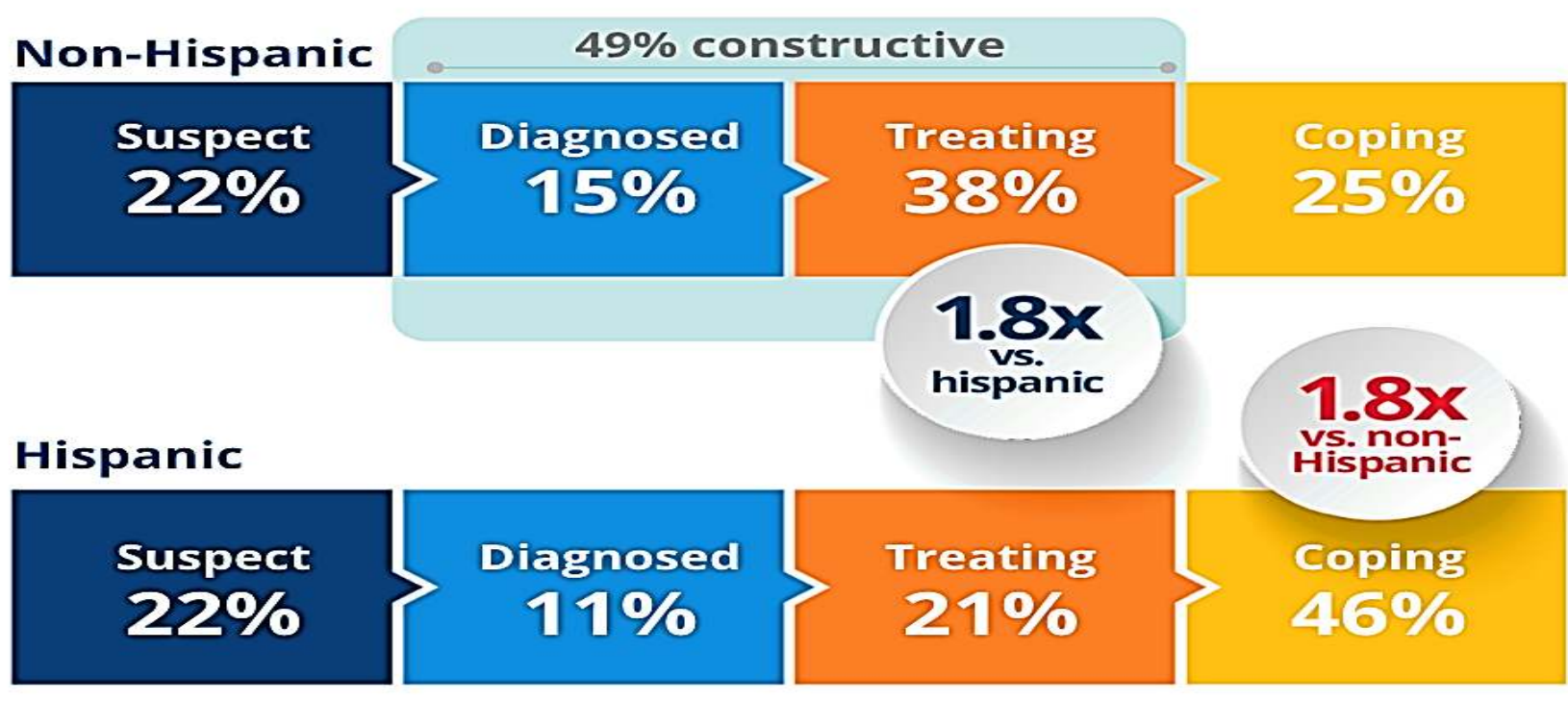

$\mathrm{n}=43 \mathrm{~K}$ discussions Hispanics vs. $543 \mathrm{~K}$ discussions overall
12 months of discussions as of May 2019

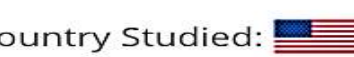

Share of Voice of Depression Discussions by Segment by Journey Stage

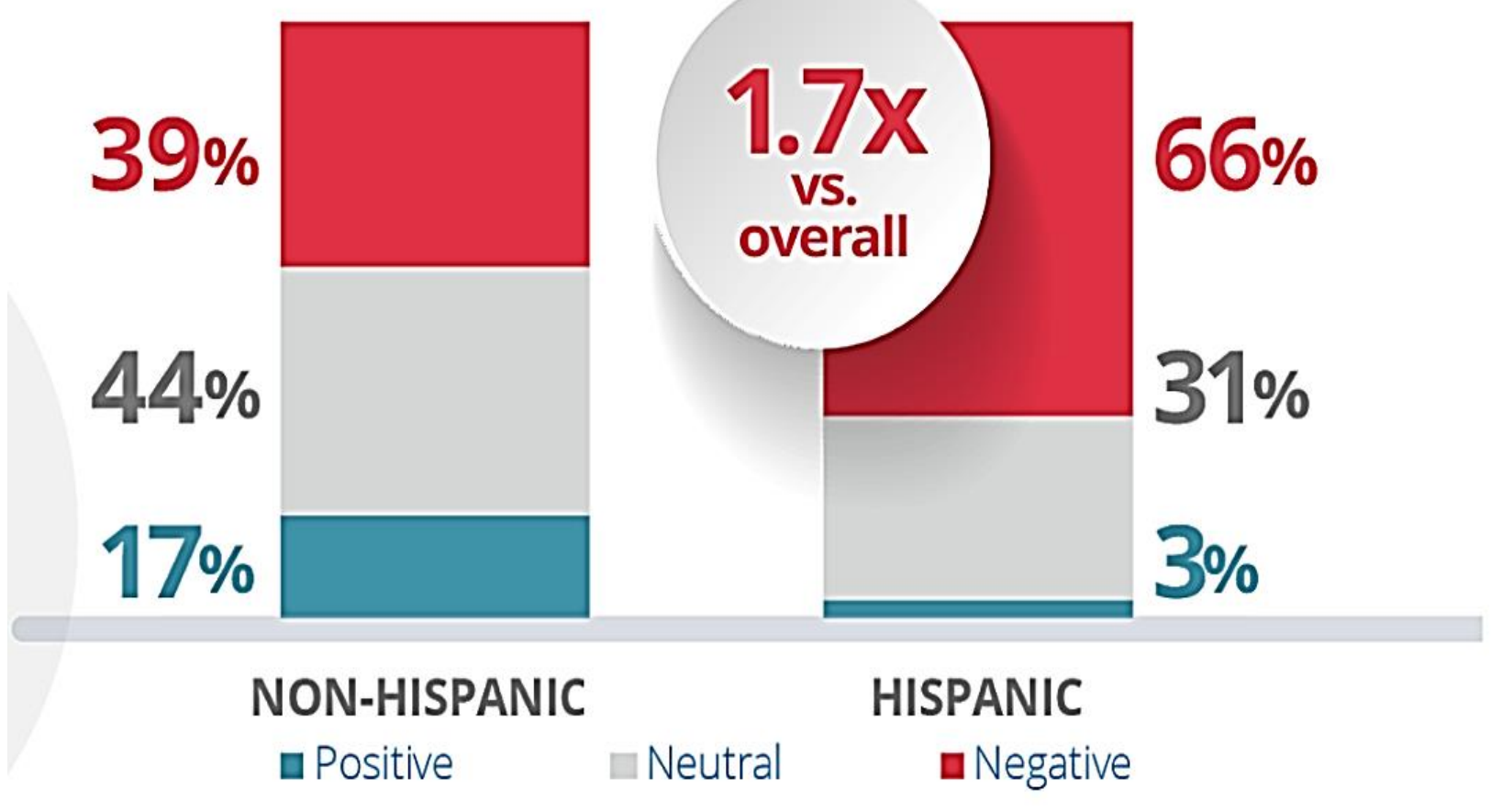

=43K discussions Hispanics vs. 398K discussions Non-Hispanics

12 months of digital discussions as of May 2019

Country Studied:

Depression Digital Conversation Sentiment Profile by Segment
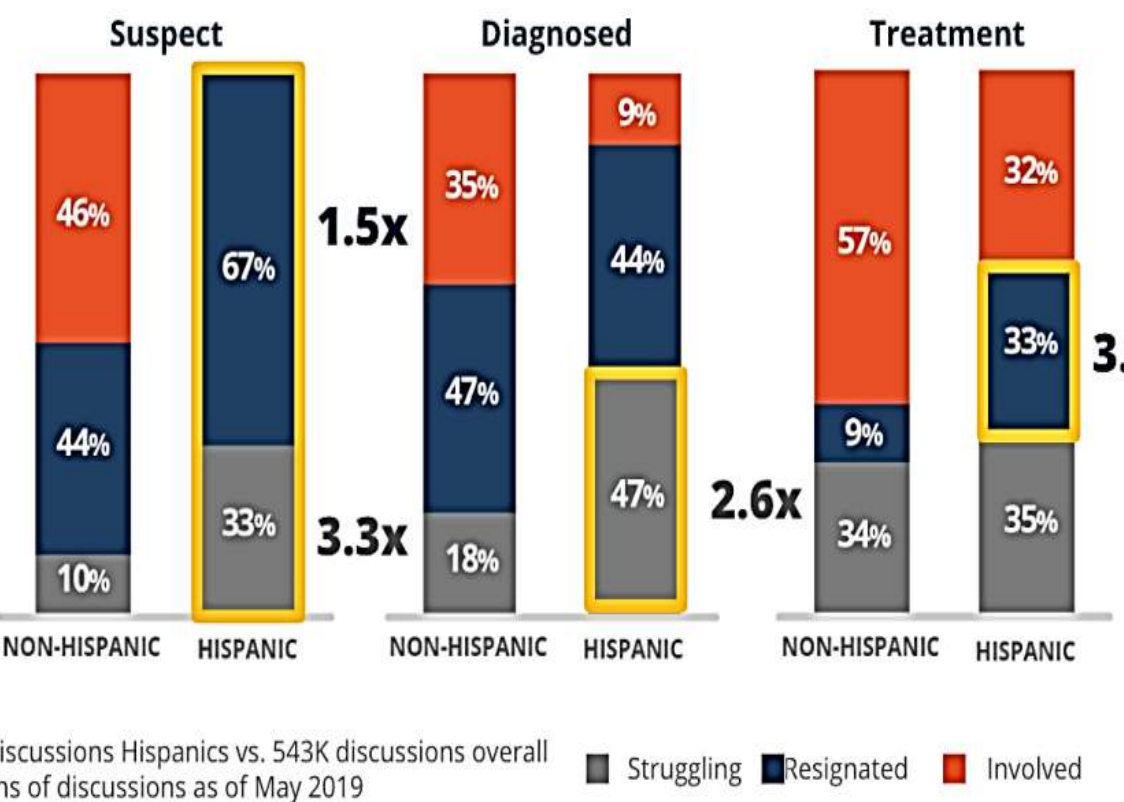

Coping

Attitude Profile by Segment by Patient Journey Stage

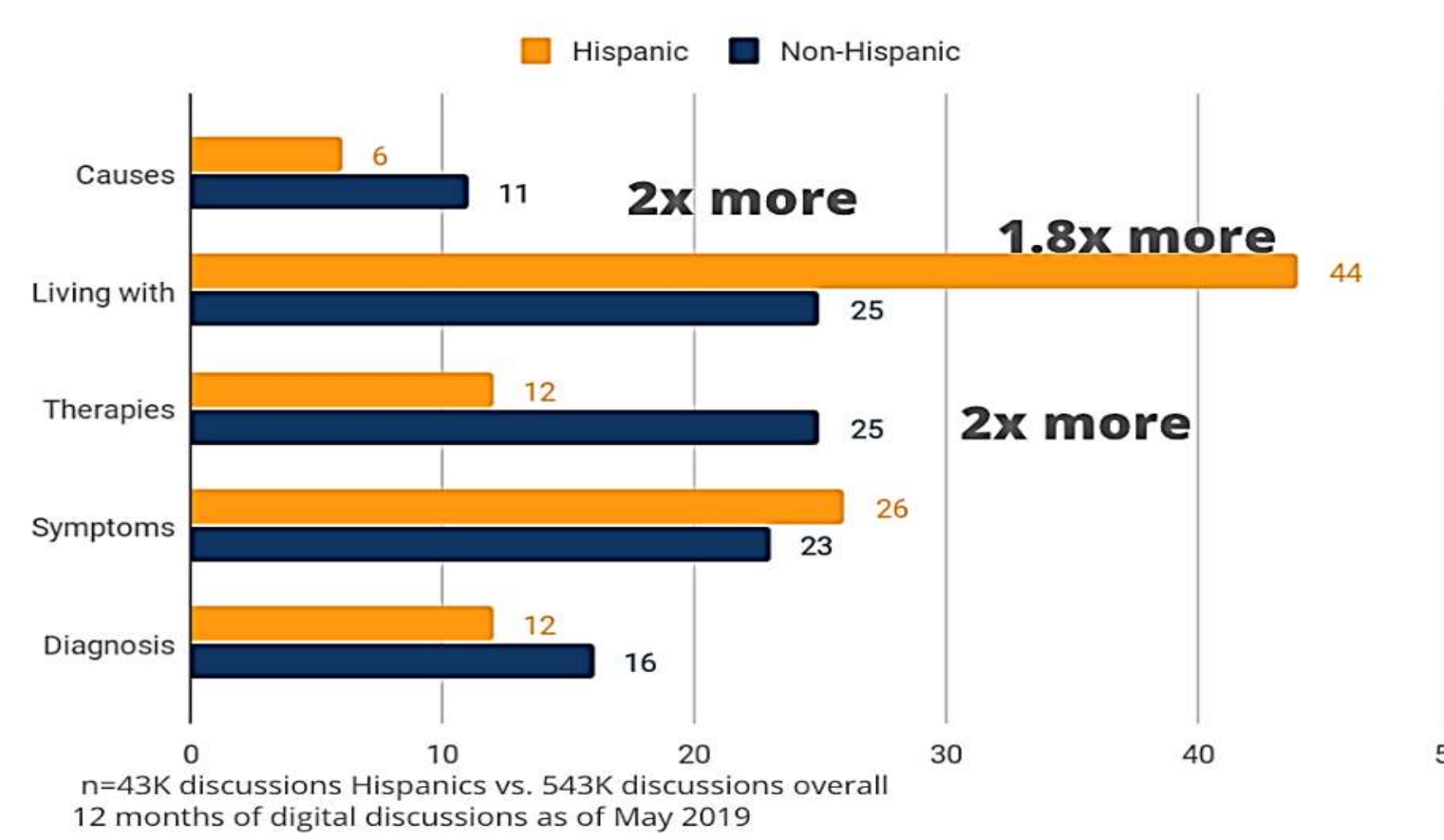

$3.7 x$

NON-HISPANIC HISPANIC

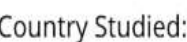

